\title{
An Evaluation of Hepatotoxicity and Nephrotoxicity of Liposomal Amphotericin B (L-AMB)
}

\author{
Gourang P. Patel • Christopher W. Crank • \\ Jerrold B. Leikin
}

Published online: 6 November 2010

(C) American College of Medical Toxicology 2010

\begin{abstract}
Hepatic and renal functions are important considerations when selecting antifungal therapy. This investigation of liposomal amphotericin B (L-AMB) was conducted to determine the incidence and factors associated with the development of hepatotoxicity and nephrotoxicity. A retrospective chart review was conducted of 100 consecutive patients receiving L-AMB at doses of 1, 3, and $5 \mathrm{mg} / \mathrm{kg}$. Hepatotoxicity was defined as an increase of bilirubin greater than $1.5 \mathrm{mg} / \mathrm{dl}$ or AST and ALT greater than three times the normal range. Nephrotoxicity was defined as an increase in serum creatinine of $0.5 \mathrm{mg} / \mathrm{dl}$ or an increase of $50 \%$ from baseline. Patients were included if they were 18 years of age or older. Patients were excluded if they had developed hepatic or renal dysfunction prior to L-AMB administration. Seventy-five patients were included based upon the predefined inclusion/exclusion criteria. Twenty-one percent (16/75) developed hepatotoxicity based upon the predefined criteria. There were no additive correlates for this adverse effect. Overall, 56\% (42/75) of
\end{abstract}

The following research has been presented at the North American Congress of Clinical Toxicology (NACCT) meeting 2009 in San Antonio, TX.

G. P. Patel $\cdot$ C. W. Crank

RUSH University Medical Center,

Chicago, IL, USA

J. B. Leikin

NorthShore University Health-System OMEGA,

Glenview, IL, USA

G. P. Patel $(\bowtie)$

Medical Intensive Care Unit, Department of Pharmacology, Division of Pulmonary and Critical Care Medicine,

RUSH University Medical Center,

1653 West Congress Parkway, Jelke 297,

Chicago, IL 60612, USA

e-mail: Gourang_p_patel@rush.edu patients developed nephrotoxicity. Seventy-four percent (31/42) were exposed to IV contrast, and 90\% (38/42) were receiving nephrotoxins concurrently. Age, cumulative dose, concomitant nephrotoxins, and IV contrast exposure were associated with increased nephrotoxicity $(p<0.001)$. The development of hepatotoxicity was observed; however, no correlates (age, dose escalation, or cumulative dose) were significantly associated with its occurrence. Overall nephrotoxicity with L-AMB was common and often multifactorial. Lipid amphotericin B products are associated with lower rates of nephrotoxicity than conventional amphotericin; however, in this analysis, L-AMB was associated with a high incidence of nephrotoxicity.

Keywords Liposomal amphotericin · Nephrotoxicity . Hepatotoxicity · Antifungal

\section{Introduction}

Systemic organ dysfunction is a common phenomenon in the intensive care unit (ICU). Hepatic and renal dysfunction in the intensive care unit has a significant impact on patient outcome and represents a substantial healthcare burden [1]. Selecting an antifungal agent requires consideration of renal and hepatic function and probable or proven site of infection. The three main classes of antifungal therapy include triazoles, polyenes, and echinocandins. These three medication classes provide coverage against yeast and/or mold infections [2-4]. One class of antifungal, polyenes, play a significant role in the management of these fungal pathogens. Lipid-based amphotericin B preparations have significantly less toxicities than conventional amphotericin B deoxycholate (AMB) [5, 6]. The three main lipid-based preparations available for amphotericin $\mathrm{B}$ (AMB) include 


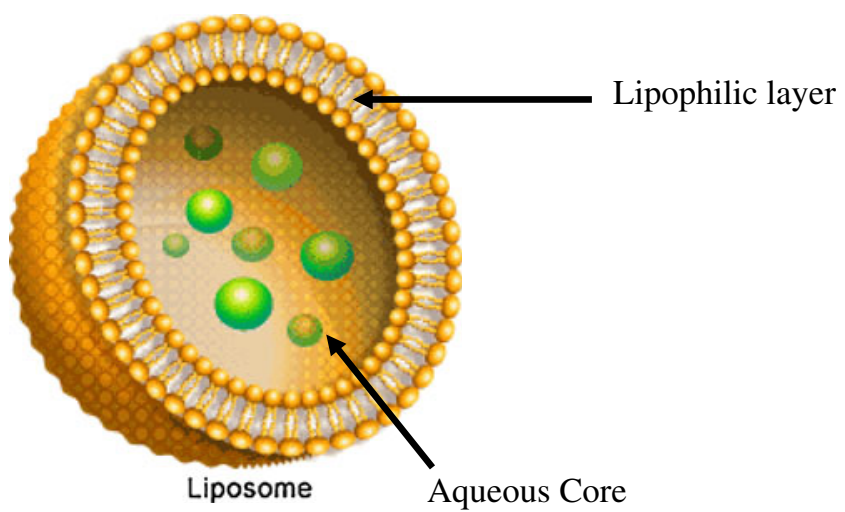

Fig. 1 Liposome drug delivery

the following: amphotericin B colloidal dispersion $(\mathrm{ABCD})$, amphotericin lipid complex (ABLC), and liposomal amphotericin B (L-AMB).

Liposome utilization for drug delivery is based on the concepts of increasing the amount of drug to the active site of activity while simultaneously decreasing toxicity. The concepts focus on targeting only tissues or organs only affected by disease, while sparing the unaffected areas of the body. Liposomes are composed of an aqueous core with one or more layers of either natural or synthetic lipids [7]. For example, medications which are more lipophilic remain in the lipid area and those which are more hydrophilic are gathered in the aqueous area of the liposome (Fig. 1). In addition, the liposome can protect the medication from degradation and are largely contained via the mononuclear phagocyte system (MPS). Once the contact is initiated with the MPS, lysis occurs, and the contents carried via the liposome are exposed [7]. Several examples of available current conventional liposomal medications include the following: doxorubicin, cisplatin, depocyt, and amphotericin [7]. This case-series investigation of liposomal amphotericin B (L-AMB) was conducted to determine the incidence and factors associated with the development of hepatotoxicity and nephrotoxicity.

\section{Materials and Methods}

The investigation was a retrospective case-series chart review that was conducted at RUSH University Medical Center in Chicago, IL. The study was approved by the Investigational Review Board (IRB) for Human Experimentation. The retrospective review was conducted of 100 consecutive patients receiving L-AMB from January 2007 through December 2007. Patients were identified by an electronic pharmacy prior drug approval database. L-AMB doses of 1 , 3 , and $5 \mathrm{mg} / \mathrm{kg}$ were evaluated. Hepatotoxicity was defined as an increase of bilirubin greater than $1.5 \mathrm{mg} / \mathrm{dl}$ or AST and ALT greater than three times the normal range. Nephrotox- icity was defined as an increase in serum creatinine of $0.5 \mathrm{mg} / \mathrm{dL}$ or an increase of $50 \%$ from baseline. Inclusion criteria included age greater than 18 years and having received L-AMB at one of the evaluated doses. Exclusion criteria included the development of hepatic or renal dysfunction prior to L-AMB administration. Baseline demographics were collected including age, gender, baseline serum creatinine, immunosuppressant regimen, intravenous (IV) contrast exposure, concomitant hepatotoxins or nephrotoxins, and length of L-AMB treatment. Examples of hepatotoxic exposures include the following: rifampin, isoniazid, and chemotherapeutic agents (i.e., cyclophosphamide, etoposide, carmustine, melphalan, cyclosporine, and tacrolimus). Examples of nephrotoxic exposures include the following: aminoglycosides, cyclosporine, and tacrolimus.

\section{Statistics}

Statistical analyses were performed using SPSS version 15 Chicago, IL. Categorical variables were analyzed utilizing the Chi-square analysis and continuous variables utilizing a $t$ test. In addition, a logistic regression was performed to evaluate the effect of multiple variables on the outcome of hepatotoxicity and nephrotoxicity. Variables were then considered for inclusion into the logistic regression model if the bivariate analyses demonstrated a $p$ value of less than $0.2[8]$.

\section{Results}

One hundred patients were initially identified from the original pharmacy database, of which 75 patients were included based upon the predefined inclusion/exclusion criteria (Table 1). Of the patients included into the review, $21 \%(16 / 75)$ developed hepatotoxicity based upon the predefined criteria. There were no additive correlates for this adverse effect. Overall, $56 \%(42 / 75)$ of patients developed nephrotoxicity and overall incidence of hepatotoxicity of $21 \%(16 / 75)$. Hematology/oncology patients comprised $79 \%(59 / 75)$ of the total population, and the majority had either hematologic, lung, or ovarian malig-

Table 1 Baseline characteristics

\begin{tabular}{lc}
\hline Demographics $(n=75)$ & Mean (SD) \\
\hline Age (years) & $49(17)$ \\
Baseline serum creatinine $(\mathrm{mg} / \mathrm{dL})$ & $0.85(0.29)$ \\
Bilirubin $(\mathrm{mg} / \mathrm{dL})$ & $0.9(0.5)$ \\
AST/ALT (U/L) & $25 / 30(10)$ \\
Total duration of treatment (days) & $9.65(6.7)$ \\
Length of stay (days) & $27(14.5)$ \\
\hline
\end{tabular}


nancy. Of the patients that developed nephrotoxicity, $74 \%$ (31/42) were also exposed to IV contrast. In addition, $90 \%$ (38/42) were receiving nephrotoxins concurrently. Examples of nephrotoxins evaluated include: cyclosporine, tacrolimus, methotrexate, aminoglycosides, and foscarnet. Age, cumulative dose, dose escalation, concomitant nephrotoxins, and IV contrast exposure were found to be independently associated with increased incidence of nephrotoxicity $(p<0.001)$ (Fig. 2). Therefore, a logistic regression analysis was performed evaluating the individual variables and nephrotoxicity. Age demonstrated to be the only significant variable to have an effect on nephrotoxicity $(p=0.007)$.

\section{Discussion}

The incidence of the three most common adverse drug events (ADRs) resulting from amphotericin B therapy can be described as infusion-related reactions, hepatotoxicity, and nephrotoxicity $[9,10]$. The following study specifically evaluated the incidence of hepatotoxicity and nephrotoxicity with the use of L-AMB formulation.

\section{Hepatotoxicity}

Elevation of liver enzymes and function tests can often be difficult to determine in a patient clinical setting. Our investigation revealed that the incidence of hepatotoxicity associated with L-AMB was $21 \%$ (16/75) based upon our predefined criteria with an unadjusted incidence of 2.2 cases per 100 patient days which was similar to the study by Fischer and colleagues [10]. Our criteria of utilizing elevation of transaminases and bilirubin have been reported by other investigators prior as a surrogate for liver function during drug therapy $[10,11]$. After controlling for concur-

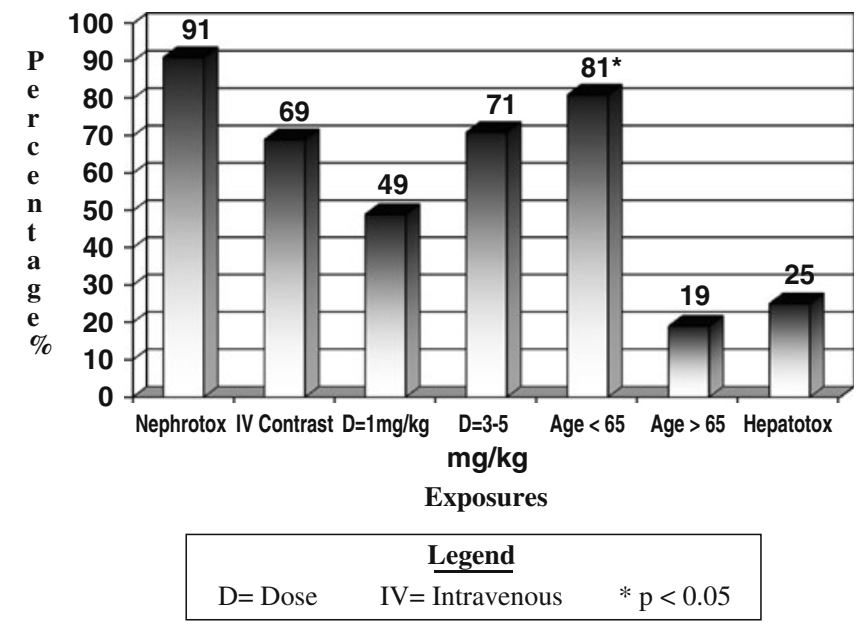

Fig. 2 Risk factors for L-AMB nephrotoxicity and hepatotoxicity rent hepatotoxic exposures (i.e., chemotherapy) there were no correlates for this adverse drug event when utilizing a multivariate logistic regression model. The package insert information for L-AMB lists an incidence of hepatotoxicity as the following: elevations of AST/ALT as $10 \%$ and bilirubin as $17 \%$ [12]. Prior investigations by Fisher and colleagues have determined that the incidence of hepatotoxicity with antifungal therapy to be approximately 0.78 cases per 100 patient days with AMB, 0.98 cases per 10 patient days for fluconazole, and 1.5 cases per 100 patient days for L-AMB [10]. The probability associated with hepatotoxicity had an OR 1.99 (95\% CI 1.21-3.26). Characteristics of the population were an elevation in serum transaminases and bilirubin. The proposed mechanism for this observation centers on the lipid component of the L-AMB. Secondary to the lipophilic nature of the drug, it has an increased affinity towards binding with biological membranes and lipoproteins. As a result of these interactions, AMB can accumulate in the liver and could lead to liver failure (i.e., elevation of transaminases and/or bilirubin) $[13,14]$.

\section{Nephrotoxicity}

Nephrotoxicity is perhaps the most described adverse drug event for AMB. Initial changes to the formulation have been able to decrease the original incidence of nephrotoxicity of approximately $50-65 \%$ [14]. Newer developments of lipid preparation have been able to reduce this figure; however, a true incidence is still difficult to determine secondary to the patient populations studied and confounders in the analyses. Considering the three formulations available, current literature ranks them in order of nephrotoxicity from highest to lowest as $\mathrm{AMB}>\mathrm{ABCD}=\mathrm{ABLC}>$ L-AMB $[14,15]$. One critique as mentioned earlier is that many of the prior evaluations had confounders in the evaluations that were unaccounted for during statistical analyses. Our investigation attempts to resolve these issues and determine that nephrotoxicity does have a higher incidence in our evaluation of $56 \%(42 / 75)$ with risk factor stratification when this adverse drug event is evaluated in clinical practice. The unadjusted incidence of nephrotoxicity was 5.8 cases per 100 patient days. The current package insert for L-AMB lists the incidence of nephrotoxicity as $20 \%$ [12].

The pathophysiology and pharmacology of nephrotoxicity from AMB has been well described. One reason the incidence was observed to be higher than typically reported is that the patient populations receiving L-AMB for probable or confirmed fungal infections can be critically ill (i.e., in the ICU) and/or have underlying disease states and exposure (i.e. cancer \pm recent chemotherapy) that can affect the presentation of nephrotoxicity. The proposed 
mechanism of AMB nephrotoxicity has been described as multifactorial. Both mechanisms described as the AMB interacting with the epithelial cell membranes within the kidney tubule $[13,14]$. Once the AMB interacts with these membranes, there are a series of reactions that trigger vasoconstriction which can lead to hypoxic conditions that translate into ischemic tubule injury. In addition, the direct exposure of AMB to the region leads to marked wasting and depletion of electrolytes (i.e., $\mathrm{K}+$ and $\mathrm{Mg} 2+$ ) and loss of cytoplasmic adenosine tri-phosphate [13]. One characteristic finding about the renal dysfunction is that it initially presents as non-oliguric renal failure (i.e., urine output greater than $400 \mathrm{ml} / 24 \mathrm{~h}$ ). One approach for prevention of nephrotoxicity has been described using hydration with $0.9 \%$ NS. The hydration would need to be completed $1 \mathrm{~h}$ prior to the administration of the medication [16]. One challenging aspect of this approach is that many of these patients are either receiving additional hydration (i.e., secondary to chemotherapy) or may be volume restricted which makes implementing this strategy difficult. The proposed mechanism for this is that a dramatic increase in initial urine output is secondary to the disruption of AVParginine vasopressin (aquaporin-2) channels [13].

There are also several limitations in the study. The first limitation was that the cohort used to define our patient population for this study was split between the intensive care unit and hematology/oncology medicine floor at 44\%/ $56 \%$, respectively. Additional limitations include that the study is single-centered and contains a small number of patients. Another limitation of the study was that to truly see the effect of L-AMB on liver and kidney function, it should be the only exposure to the patient. However, patients that would require L-AMB therapy are acutely ill patients; therefore, exposure to additional nephrotoxins and heptotoxins often has already been administered as well.

The patient population that are receiving antifungal therapy often have underlying disease process (i.e., cancer) and/or exposure to risk factors (i.e., medications, intravenous contrast) for the development of hepatotoxicity and nephrotoxicity. Based on these considerations, it is difficult to truly report ADRs for L-AMB; however, it appears that both toxic drug effects (i.e., nephrotoxicity and hepatotoxicity) have the potential to develop, and precautions should be taken including pre-medication, hydration $(0.9 \% \mathrm{NS})$, and increased pharmacovigilance. In our investigation, the development of hepatotoxicity was observed; however, no correlates (age, dose escalation, or cumulative dose) were significantly associated with its occurrence. Overall, nephrotoxicity with L-AMB was common and often multifactorial. Clinicians are encouraged to have an awareness for nephrotoxicity and hepatotoxicity with the use of L-AMB therapy. In addition, some measures can be taken to reduce nephrotoxicity (i.e., saline hydration, lower dosing $3-5 \mathrm{mg} / \mathrm{kg}$, and exposures to additional nephrotoxins) and hepatotoxicity (i.e., limiting exposures to additional hepatotoxins).

Disclosure of Potential Conflict of Interest The authors have no disclosures.

\section{References}

1. Martin CM, Hill AD, Burns K, Chen LM (2005) Characteristics and outcomes for critically ill patients with prolonged intensive care unit stays. Crit Care Med 33:1922-1927

2. Pappas PG, Kauffman CA, Andes D et al (2009) Clinical practice guidelines for the management of candidiasis: 2009 update by the infectious disease society of America. Clin Infect Dis 48:503-535

3. Walsh TJ, Anaissie EJ, Denning DW et al (2008) Treatment of aspergillosis: clinical practice guidelines of the infectious disease society of America. Clin Infect Dis 46:327-360

4. Gueret R, Patel GP, Simon DM, Balk RA (2007) Invasive aspergillosis: case report and review of the approach to diagnosis and treatment. Clin Pulm Med 14:197-205

5. Tiphine M, Letscher-Bru V, Herbrecht R (1999) Amphotericin B and its new formulations: pharmacologic characteristics, clinical efficacy, and tolerability. Transpl Infect Dis 1:273-283

6. Dupont B (2002) Overview of the lipid formulations of amphotericin B. J Antimicrob Chemother 49(s1):31-36

7. Immordino ML, Dosio F, Cattel L (2006) Stealth liposomes: review of the basic science, rationale, and clinical applications, existing and potential. Int J Nanomedicine 1:297-315

8. Harrell FE Jr, Lee KL, Matchar DB et al (1985) Regression models for prognostic prediction: advantages, problems, and suggested solutions. Cancer Treat Rep 69:1071-1077

9. Roden MM, Nelson LD, Knudsen TA et al (2003) Triad of acute infusion-related reactions with liposomal amphotericin B: analysis of clinical and epidemiological characteristics. Clin Infect Dis 36:1213-20

10. Fischer MA, Wolfgang WC, Rubin RH, Avorn J (2005) The hepatotoxicity of antifungal medications in bone marrow transplant recipients. Clin Infect Dis 41:301-307

11. Floyd J, Mirza I, Sachs B, Perry MC (2006) Hepatotoxicity of chemotherapy. Semin Oncol 33:50-67

12. AmBisome package insert. Astellas Pharma. Revised:April 2005

13. Lemke A, Kiderlen AF, Kayser O (2005) Amphotericin B. Appl Microbiol Biotechnol 68:151-162

14. Deray G (2002) Amphotericin B nephrotoxicity. J Antimicrob Chemother 49(s1):37-41

15. Ullmann AJ, Sanz MA, Tramarin A et al (2006) Prospective study of amphotericin B formulations in immunocompromised patients in 4 European countries. Clin Infect Dis 43:29-38

16. Llanos A, Cieza J, Bernardo J et al (1991) Effect of salt supplementation on amphotericin B nephrotoxicity. Kidney Int 40:302-308 